\title{
Testing the Determinism of EEG and MEG
}

\author{
Werner Mühlnickel, ${ }^{1}$ Nicola Rendtorff, ${ }^{2}$ Zbigniew J. Kowalik, 1,2 \\ BrigitTe Rockstroh, ${ }^{2}$ Wolfgang Miltner, ${ }^{3}$ and Thomas Elbert ${ }^{1}$ \\ ${ }^{1}$ Institute for Experimental Audiology, University of Münster \\ ${ }^{2}$ Department of Clinical Psychology, University of Konstanz \& Neurological Rehabilitation Unit, \\ Kliniken Schmieder, Allensbach, and \\ ${ }^{3}$ Department of Medical Psychology, University of Tübingen
}

\section{Introduction}

Neural MASS ACTIVITY produces irregular time series such as the EEG and MEG. It is already apparent, through visual inspection, that these time series cannot simply result from an uncoordinated arbitrary firing of neurons. Indeed, we expect that neurons must cooperate and partially synchronize their firing patterns in order to produce meaningful output. Although we may believe that we have visually identified patterns within these irregular time series, attempts to systematically track the code with linear and stochastic statistical techniques have left us in frustration.

More recently it has been suggested that the ability to trace the dynamics of a system (e.g., to freeze them in a state space, a space that is spanned by the system's variables) might decode more of the brain's cryptic and enigmatic language (Elbert, et al., 1994). If the dynamics of the underlying system can be reduced to a set of deterministic laws, then the phase space trajectory will converge toward a subset of the phase-space. This invariant subset is referred to as an attractor. Given a particular time series, the initial question one may ask is if one can identify an attractor. If the answer is yes, then it is possible to view the series as a manifestation of a deterministic dynamic system (albeit possibly a very complex one).

How can we gain information about the deterministic processes governing a particular nonlinear system? As a first step, it is possible to estimate the determinism inherent in a given time series. Kaplan \& Glass (1992) developed a direct test for determinism in a given time series. This article introduces this method for analyzing EEG and MEG and presents comparisons with other nonlinear measures such as the fractal dimension. The estimations of the fractal dimension of the EEG has received considerable attention starting with the studies of Agnes Babloyantz (Babloyantz, 1985; Babloyantz \& Destexhe, 1986; Babloyantz, et al., 1985. See Elbert, et al., 1994, for a recent review). In principle, such an estimation of the fractal dimension of the system generating a time series requires a "sufficient number" of data points. Estimations of d-dimensional systems reach up to more than $10^{\mathrm{d}}$ points. When dimensions are estimated from physiological time series containing less than 10,000-20,000 points, the resulting values cannot be accepted as absolute terms. Results can only bè interpreted when comparisons between different time series are made, such as those between conditions or groups. Furthermore, data are nonstationary, and the measures presume that the data generator does not change. Therefore, relative dif-

Address for correspondence: Thomas Elbert, University of Münster, Kardinal-von-Galen-Ring 10, D-48129 Münster, Germany. 
ferences in dimension between conditions, or within the same condition, may be of more practical interest than any inaccurate estimate of the absolute value.

More recently, surrogate data and statistical null hypotheses testing has been used to examine whether a given time series can be considered different from that of white or colored noise. This is important as Osborne \& Provenzale (1989) demonstrated that colored random noise, if analyzed by the Grassberger-Procaccia procedure, may give finite and predictable values of the correlation dimension. Such a result would suggest the presence of a chaotic system, when, in actuality, only noise existed.

At this time there remain a number of technical issues as to what the best type of control signal might be for comparisons with EEG analysis. Lutzenberger, et al. (1993) used, as a control signal, computer-generated random series that were filtered to give power spectra identical to the individual EEG traces. Pjin, et al. (1991) used a procedure based on an earlier paper of Theiler to compare the EEG from the rat limbic cortex with that of a control signal constructed from the same EEG: Theiler, et al. (1992) suggest two separate algorithms for generating surrogate data. The procedure determines the Fourier transform of the original data set, randomizes the phases of this transform, and then produces a second data set by taking the inverse transform. By applying Grassberger-Procaccia to both data sets, it is possible to determine statistically if a difference between the two data sets exists. In this way, one can evaluate if the actual data set is more than linearly correlated noise. This type of procedure implemented by Pritchard \& Duke (in press) for human EEG data has the additional advantage of establishing an inferential probability in the testing of the null hypothesis (i.e., the signal is not different from that of noise) as well as being less difficult. It has numerous advantages over making the claim that one is measuring dimensionality directly in a particular real world signal. Additionally, if the null hypothesis cannot be rejected, there will be no advantage in determining a particular measure of dimensionality.

\section{The Deterministic Test of a Time Series ${ }^{1}$}

The goal of the presently introduced method for EEG/MEG analyses is to obtain information about the determinism of a time series, even for a short-time interval of measurement. Kaplan \& Glass (1992) developed a direct test for determinism in a time series. This method is based on the fact that the tangent of the trajectory of a deterministic system is a function of the region in the phase space. All tangents in a given region of the phase space have nearly the same orientation.

The estimation of the determinism is briefly described in the following steps:

- If the attractor is embedded in a d-dimensional phase space, then the phase space is divided into $\mathrm{n}^{\mathrm{d}}$ boxes (where $\mathrm{n}$ is an integer).

- If a trajectory passes through a box $\mathrm{j}$, then a vector $v_{k j}$ ( $\mathrm{k}$ pass) is constructed. This vector is defined by the coordinates of the starting-point and the end-point of that part of the trajectory inside box $\mathrm{j}$.

- The sum of all $v_{k j}$ yields a vector $V_{j}=\frac{1}{n_{j}} \sum_{k} v_{k j}$. The $V_{j}$ characterizes the attractor.

- The average $\bar{L}_{n}^{d}=<\left\|V_{j}\right\|>n_{j=n}$ over earh box containing n passes shows a statistic over all $V j$ with $\mathrm{n}$ passes. 
$\bar{L}_{n}^{d}=1$ is valid only for deterministic systems with infinite time series and a box edge length $\rightarrow 0$. White noise falls off with $\bar{L}_{n}^{d}=n^{-\frac{1}{2}}$ (Kaplan \& Glass, 1992).

This method was applied to EEG and to MEG signals as outlined below.

\section{Comparisons Between EEG- and MEG-time Series With Filtered Noise and Time Series Constructed by Means of Well-known Nonlinear System Equations}

EEG and MEG signals were recorded from an epileptic patient and a control subject during a relaxed waking state with eyes open. MEG was measured from 37 positions over the right temporal areas and was sampled with a frequency of $298 \mathrm{~Hz}$. Furthermore, the output of the MEG-system was measured using the same settings when no subject was present, i.e., only noise of the system. Random numbers generated by a standard algorithm were added with both inputs having equal signal power.

The times slices analyzed contained 3,000 points. The reconstructed trajectories consisted of a limited number of points, thus the statistic $L(d, n)=\bar{L}_{n}^{d}$ was replaced by a dynamic version $L(d, n)=\overline{L D}_{n}^{d}$. The static version estimates $L(d, n)=\overline{L D}_{n}^{d}$ after the complete reconstruction of the attractor. If, for example, the number of passes is $n=5$, then in $L(d, 5)$ there would be boxes with $n=5$ passes. Another possibility would be to consider boxes that could contain more than $\mathrm{n}=5$ passes, meaning that the sum vector $V_{j}$ was calculated subsequently for each pass. If one given box was passed $n=20$ times, then this box was considered for each pass $\mathrm{n}$ up to $\mathrm{n}=20$. The result for $L D(d, n)$ shows a gain of deterministic patterns in a system.

Table 1 compares the statistics $\bar{L}_{n}^{d}$ and $\bar{L} \bar{D}_{n}^{d}$ for an embedding dimension of d=7 and a delay time of $\tau=5$. The presented values were averages of the interval $u=[11,20]$ passes. Each time series contains 3,000 data points and their autocorrelation functions are identical.

Table 1 includes results from time series of corresponding length generated by (a) the Lorenz attractor (b) the van-der-Pol-like oscillator, ${ }^{2}$ (c) the Glass-Mackey equations with the results for (d) the noise and (e) the EEG and MEG-recordings measured over central locations $\left(\mathrm{C}_{4}\right)$. Two important outcomes are obvious: (1) a higher deterministic value for the MEG and for the EEG than for the white noise and (2) an increase in the dynamic patterns in a system in the dynamic version $\left(\overline{L D}_{n}^{d}\right)$. Furthermore, the results for the epileptic patient were even higher than those for a control subject, an observation that is in agreement with the literature (Elbert, et al., 1994).

MEG-analyses tend to produce higher values for determinism than EEG-based computations, a result that confirms the higher specificity of MEG. ${ }^{3}$

\section{Example of a Clinical Study: EEG-determinicity in Healthy Controls and In Patients With Coma and Apallic Syndrome}

The main goal of the present study was to evaluate the ability of the determinism measure to gain relevant clinical information; particularly, information other than the EEG 
TABLE 1. Comparisons for the static and the dynamic version of the test for determinism.

\begin{tabular}{|c|c|c|}
\hline & Static $\bar{L}_{n}^{d}$ & Dynamic $\overline{L D}_{n}^{d}$ \\
\hline Lorenz & $.943 \pm .001$ & $.977 \pm .001$ \\
\hline van der Pol-type & $.601 \pm .010$ & $.779 \pm 007$ \\
\hline Glass-Mackey & $.542 \pm .002$ & $.545 \pm 001$ \\
\hline MEG (epileptic p.) & $338 \pm .021$ & $.389 \pm .001$ \\
\hline EEG (epileptic p.) & $.402 \pm .030$ & $.378 \pm 001$ \\
\hline MEG (control) & $.365 \pm .002$ & $.381 \pm .001$ \\
\hline filtered noise & $.300 \pm .005$ & $.303 \pm .002$ \\
\hline
\end{tabular}

power spectra. For this purpose, we related characteristics of the EEG of patients with apallic syndrome with their clinical condition and compared the EEG measures to that of healthy control subjects.

The apallic syndrome (also termed persistent vegetative state, Jennet and Plum, 1972) results from closed-head-injury or severe CNS trauma with functional disturbance of the brain stem that lead to a separation of cortical functions from brain stem functions (see Rendtorff et al., in prep for further description of state and sample). For prognostic evaluation as well as for the selection of appropriate treatment, it is important to assess the severity of CNS dysfunction and to detect changes in condition. So far, diagnosis and prognosis are based on clinical evaluation, supported by rating scales such as the Disability Rating Scale (DRS, Rappaport et al., 1982). Few studies have utilized EEG as an additional objective measure for diagnostic and prognostic purposes. Zeitlhofer et al. (1991) could not detect any prognostic value of auditory or somatosensory-evoked potentials for the prognosis of apallic patients.

In the present study, spontaneous EEG activity recorded without any specific external stimulation were evaluated on the basis of nonlinear measures and the conventional power spectrum analysis. The characteristics of the EEG data (recorded from patients with apallic syndrome) were compared to the respective characteristics of the EEG of both coma patients and healthy subjects.

Sample: The EEG was monitored in 13 patients ( 8 male, 5 female) with apallic syndrome resulting from hypoxia $(\mathrm{N}=6)$, polytrauma $(\mathrm{N}=5)$ or subdural hemorhage $(\mathrm{N}=2)$. Patients were excluded who (1) required artificial ventilation (2) were medically unstable (3) exhibited massive abnormalities in spontaneous EEG or (4) did not exhibit normal 
brain stem auditory-evoked potentials (BAEPs). The depth of coma was documented by the DRS. The mean score on the DRS score upon admission was 22.4 (range 20-24), which corresponds to severe disability. ${ }^{4}$ EEG-recordings were obtained six times (always at the same time of day) with one week intervals.

The EEG from seven student volunteers ( 4 male, 3 female, mean age 26.8 years, range 24-31) served for purposes of comparison. EEG was recorded during two segments of 3 minutes each, taken 45 minutes apart. There was no additional task or stimulation. Control subjects participated in three sessions (once a week for 3 weeks).

In all subjects the $E E G$ was recorded along the midsagittal line $(\mathrm{Fz}, \mathrm{Cz}, \mathrm{Pz})$ with a 5 -sec time constant. The reference electrode was affixed to the right earlobe. The lowpass-filter was set at $30 \mathrm{~Hz}$. Nonpolarizable silver/silverchloride electrodes (ZAK) were used; GRASS EC2 electrolyte served as a conducting agent (electrode impedance below 5 $\mathrm{kOhm}$ ). Eye movements were monitored via $\mathrm{AgAgCl}$ electrodes affixed about $1 \mathrm{~cm}$ above and below the subject's right eye. EEG and EOG were sampled at a rate of $200 \mathrm{~Hz}$ for the 3-min recording intervals.

\section{Data Reduction and Analysis}

For every 3-min recording six segments free of artefacts and $10 \mathrm{~s}$ in length were selected. A power spectrum estimate and the deterministic measure were computed for every segment.

The deterministic measure was determined for every 2,000 pt. segment using an embedding dimension of 12 , an average for the interval $n=[10,15]$. Values in the deterministic measure, which fell below the one of correspondingly filtered and processed white noise, were excluded from further analysis. The procedure yields an average value of 0.28487 for white noise. The median was computed across the remaining values of the six original series. For a comparison between groups, values were averaged across the first three sessions. For one control subject, $80 \%$ of the values at $\mathrm{Cz}$ were missing and, therefore, the respective $\mathrm{Cz}$-value was treated as a missing value. For the purpose of correlations with the clinical scores, values of patients were averaged across all six sessions. Analyses of variance served to evaluate differences statistically.

\section{Results}

\section{, EEG Power Spectrum}

Patients produced more power in the slower frequency range (delta band) compared to controls, while there were no obvious differences between groups for other frequency bands (interaction GROUP $x$ FREQUENCY BAND, $F(4,17)=5.2, p<.05$, GROUPS, $F(1,17)=7.4, p<.01)$.

\section{Determinism}

As indicated for the central electrode by Figure 1, patients with apallic syndrome exhibited higher deterministicity than control subjects $(F(1,16)=11.5, p<.01)$. This effect is strongest at the frontal electrodes (for the difference at $\mathrm{Fz}, \mathrm{t}(18)=3.8, \mathrm{p}<.01$ ) but hardly present over posterior regions. More than half of the patients produced values of determinism above the range of control subjects. 


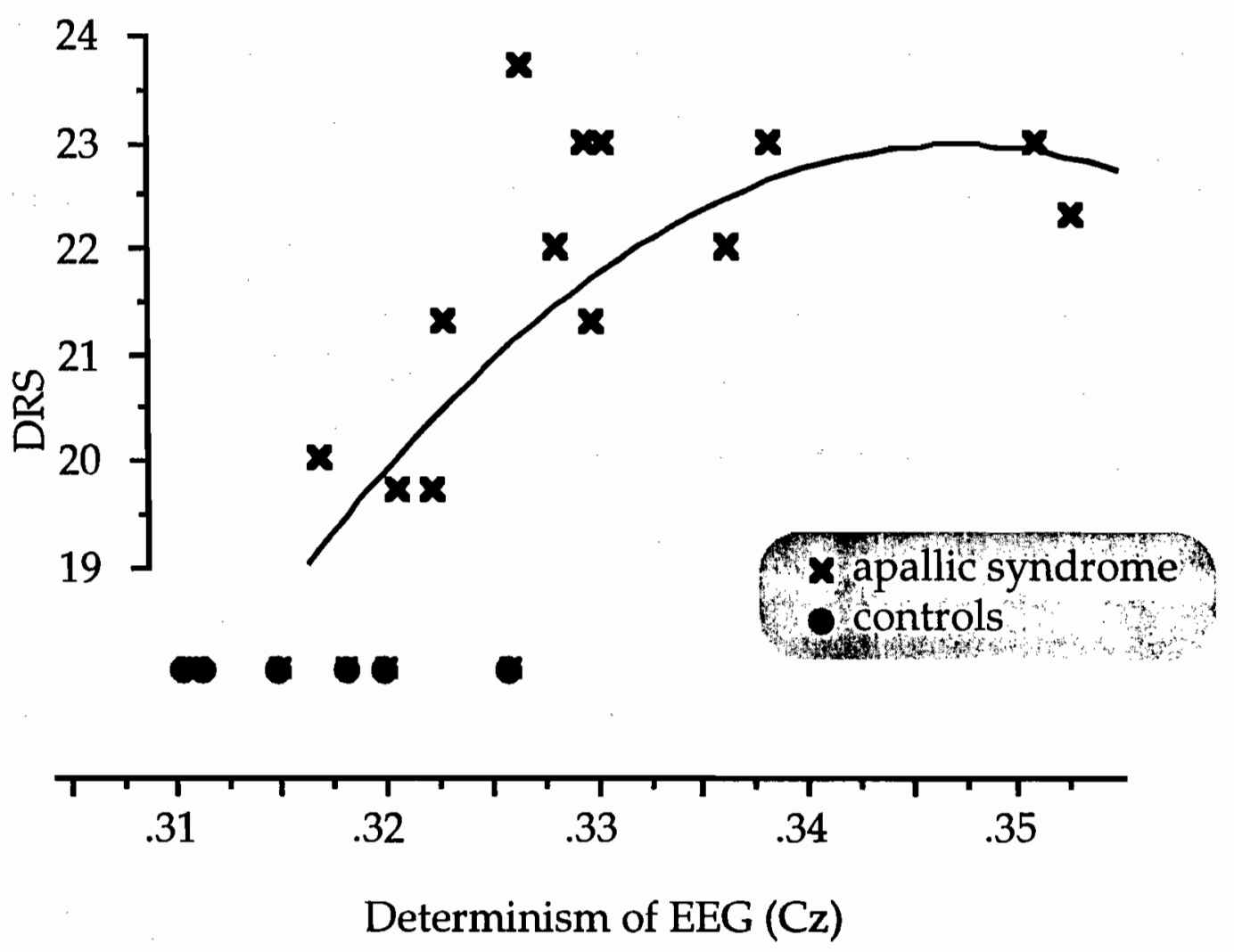

Fig. 1. Scatterplot for the determinism, averaged across sessions (1-3 for controls and 1-6 for patients.)

The severity of the comatose state (as evaluated by the DRS) correlated with the determinism determined as the average value at the $\mathrm{Cz}$-electrode across the six sessions (Figure 1): patients with higher DRS-scores (indicating more impairment) upon admission had higher scores of determinism $(\mathrm{r}=.57, \mathrm{p}<.05)$. No such relationship was found for $\mathrm{Fz}$ or $\mathrm{Pz}$. Hypoxia did not produce a clear relationship with any of the variables.

\section{Comparison With White Noise}

A comparable analysis for the white noise produces a value of 0.28487 . This value is significantly lower $(p<.001)$ than the values of each of the EEG-measurements.

\section{Other Measures of the EEG-dynamics}

Other dynamic measures, computed for exactly the same time segments, are reported in detail in Rockstroh, et al. (in press). Patients exhibited a significantly lower degree of EEG complexity than control subjects according to the PD2 (Skinner, et al., 1990), the D2 (Grassberger \& Procaccia, 1983), the mutual information function (Fraser, 1986) and the entropy (Kumpf, et al., in press) (see Table 2). None of these measures produced a significant difference between recording sites $(\mathrm{Fz}, \mathrm{Cz}, \mathrm{Pz})$. 
TABLE 2. Comparisons between patients and controls in the electroencephalographically recorded dynamic measures of brain activity. Statistically significant differences are indicated by the asterisks $\left(^{* *} p=0.01\right.$, $\left.{ }^{*} \mathrm{p}=0.05\right)$.

\begin{tabular}{llll} 
& Patients & Controls & $\mathrm{F}(1,1)$ \\
\hline Power in the EEG-bands & & & \\
Deltaband & $.114 \pm .027$ & $.089 \pm .018$ & $7.4^{*}$ \\
Thetaband & $.025 \pm .010$ & $.023 \pm .007$ & n.s. \\
Alphaband & $.011 \pm .007$ & $.014 \pm .003$ & n.s. \\
Betaband & $.003 \pm .003$ & $.007 \pm .002$ & n.s. \\
Gammaband & $.001 \pm .0001$ & $.001 \pm .0001$ & n.s.
\end{tabular}

nonlinear measures:

$\begin{array}{lrrr}\text { Determinism } & .334 \pm .012 & .32 \pm .008 & 11.5^{* *} \\ \text { PD2 } & 5.6 \pm 0.7 & 6.4 \pm 0.2 & 11.0^{* *} \\ \text { D2 (Grassberger) } & 7.6 \pm 2.8 & 9.0 \pm 2.5 & 7.3^{*} \\ \text { Mutual Info. } & -55.3 \pm 28.3 & -24.0 \pm 8.3 & 10.3^{* *} \\ \text { Kentropy5 } & -527 \pm 152 & -420 \pm 59 & 4.2^{*}\end{array}$

\section{Discussion}

In all cases MEG and EEG time series produce higher values for the determinism than the analyses of white noise of equal length, filtered and sampled in a comparable manner. This is true for all locations and for all subjects irrespective of whether measurements were taken during task performance, resting wakefulness or in a comatose state. Obviously, EEG and MEG are not simply colored noise arising from arbitrary summation of uncoordinated activity. This result is in agreement with Pritchard \& Duke (in press) who have reached the conclusion that the normal resting EEG is nonlinear and therefore not a linear-stochastic system. Furthermore, their result indicates that the resting human EEG is high dimensional and does not represent low-dimensional chaos. Therefore, computations of the fractal dimension of waking EEG cannot be theoretically justified. While it may be difficult to apply the powerful background of nonlinear system analysis for the interpretation of D2 results, such computations can produce interesting differences that may not be uncovered by other types of analyses (Elbert et al., 1994).

One example of this is provided by the present clinical study: patients with appallic syndrome exhibit higher values of determinism, the more so the more severe their disability ratings. The relationship seems very consistent, although it must be limited by the reliability of the DRS score, and probably also by its validity. It seems worthwhile to investigate further whether or not nonlinear measures may prove useful not only in diagnosis but also in the prognosis of comatose states. 


\section{Acknowledgment}

Research was supported by the Deutsche Forschungsgemeinschaft and the Kuratorium ZNS.

\section{Notes}

1. A computer program (C/Unix) is available from the authors upon request via e-mail: zjk@bifurc.unimuenster.de.

2. The Van der Pol like driven oscillator was implemented following Kowalik, et al. (in press).

3. Higher specificity of MEG is to be expected, as the influence of volume conduction is much greater for the EEG, and, furthermore, in addition to tangentially oriented current dipoles, radial sources affect the EEG but hardly MEG-recordings.

4. Usually, scores between 20 and 25 are found for apallic patients, higher scores are only obtained during acute coma.

5. Entropy is a measure of complexity that reflects loss of information or inversely the amount of information needed to describe the future state of a system. The more complex the system (with noise being the most complex) the greater the entropy.

\section{References}

Babloyantz, A. (1985). Strange attractors in the dynamics of brain activity. Berlin: Springer, 126-122.

Babloyantz, A. and Destexhe, A. (1986). Low dimensional chaos in an instance of epilepsy. Proc. Natl. Acad. USA, 83, 3513-3517.

Babloyantz, A., Salazar, J.M. and Nicolis, C. (1985). Evidence of chaotic dynamics of brain activity during the sleep cycle. Phys. Lett., 111A, 3, 152-156.

Elbert, T., Ray, W.J., Kowalik, Z.J., Skinner, J.E., Graf, K.E., and Birbaumer, N. (1994). Chaos and physiology-Deterministic Chaos in excitable cell assemblies. Physiological Reviews, 74, 1-47.

Fraser, A.M. (1986). Using mutual information to estimate metric entropy. Berlin, Heidelberg, New York: Springer 82-91.

Grassberger, P. and Procaccia, I. (1983). Measuring the strangeness of strange attractors. Physica, 9D, 183208.

Kaplan, D., and Glass, L. (1992). Direct test for determinism in a time series. Physical Review Letters, 68, $427-430$.

Kowalik, Z.J., Goerke, N., Bode, M., Purwins, H.G. Experimental study of a driven Van der Pol like oscillator. Submitted for publication.

Kumpf, K., Kowalik, Z.J., Braun, Ch., Miltner, W. Entropy and Brain Dynamics (submitted for publication).

Lutzenberger, W., Elbert, T., Ray, W.J., Birbaumer, N. (1993). The scalp distribution of the fractal dimension of the EEG and its variation with mental tasks. Brain Topography, 5, 27-34.

Osborne, A.R., Provenzale, A. (1989). A finite correlation dimension for stochastic systems with power-law spectra. Physica D, 35, 357-381.

Pjin, J.P., Van Neerven, J., Noestt, A., Lopes da Silva, F.H. (1991). Chaos or noise in EEG signals; dependence on state and brain site. Electroencephalography \& Clinical Neurophysiology, 79, 371-381.

Pritchard, W.S. and Duke, D.W. Dimensional analysis of resting human EEG II: Surrogate-Data Testing indicates nonlinearity but not low-dimensional chaos (in press).

Rockstroh, B., Miltner, W., Braun, C., Kumpf, K., Rendtorff, N., Schönle, P.W. Dynamic aspects of the EEG in patients with Apallic syndrome (submitted for publication).

Skinner, J.E., Goldberger, A.L., Mayer-Kress, G. and Ideker, R.E. (1990). Chaos in the heart: implications for clinical cardiology. Biotechnology, 8, 1018-1033.

Smith, L.A. (1988). Intrinsic limits on dimension calculations. Phys. Lett., A133, 283-288.

Theiler, J., Galdrikian, B., Longtin, A., Eubank, S. and Farmer, J.D. (1992). Using surrogate data to detect nonline arity in time series. Addison-Wesley, SFI Studies of Complexity, Proc. Vol. XII, 163-188.

Zeithofer, J., Steiner, M., Oder, W., Obergottsberger, S., Mayr, N. and Deecke, L. (1991). [The prognostic value of evoked potentials in early neurologic rehabilitation of patients with the apallic syndrome]. Z. EEG EMG (Z Elektroenzephalogr. Elektromyogr. Verwandte Geb.), 22, $10-4$. 